Check for updates

Cite this: Chem. Sci., 2019, 10, 3746

๑ All publication charges for this article have been paid for by the Royal Society of Chemistry

\section{Revising the measurement process in the variational quantum eigensolver: is it possible to reduce the number of separately measured operators? $\dagger$}

\begin{abstract}
Artur F. Izmaylov, (D) *ab Tzu-Ching Yen ${ }^{\text {ab }}$ and Ilya G. Ryabinkin (D)
Current implementations of the Variational Quantum Eigensolver (VQE) technique for solving the electronic structure problem involve splitting the system qubit Hamiltonian into parts whose elements commute within their single qubit subspaces. The number of such parts rapidly grows with the size of the molecule. This increases the computational cost and can increase uncertainty in the measurement of the energy expectation value because elements from different parts need to be measured independently. To address this problem we introduce a more efficient partitioning of the qubit Hamiltonian using fewer parts that need to be measured separately. The new partitioning scheme is based on two ideas: (1) grouping terms into parts whose eigenstates have a single-qubit product structure, and (2) devising multi-qubit unitary transformations for the Hamiltonian or its parts to produce less entangled operators. The first condition allows the new parts to be measured in the number of involved qubit consequential one-particle measurements. Advantages of the new partitioning scheme resulting in severalfold reduction of separately measured terms are illustrated with regard to the $\mathrm{H}_{2}$ and $\mathrm{LiH}$ problems.
\end{abstract}

Received 14th December 2018 Accepted 11th February 2019

DOI: $10.1039 / \mathrm{c} 8 \mathrm{sc} 05592 \mathrm{k}$

rsc.li/chemical-science on several quantum computers and used for few small molecules up to $\mathrm{BeH}_{2} \cdot{ }^{13}$

One of the big problems of the VQE is that to calculate $E_{U}$, the quantum computer measures parts of $H_{\mathrm{q}}$ rather than the whole $H_{\mathrm{q}}$ on the $U\left|\Psi_{0}\right\rangle$ wavefunction. This stems from technological restrictions of what can be currently measured on available architectures. Dramatic consequences of this restriction can be easily understood with the following simple example. Let us assume that $\hat{H}_{\mathrm{q}}=\hat{A}+\hat{B}$, where $\hat{A}$ and $\hat{B}$ are measurable components of $\hat{H}_{\mathrm{q}}$ and $[\hat{A}, \hat{B}] \neq 0$, otherwise they could be measured at the same time at least in principle. The actual hardware restrictions on measurable components are somewhat different and will be discussed later, for this illustration these differences are not important. Even if one has an exact eigenstate of $\hat{H}_{\mathrm{q}}, U\left|\Psi_{0}\right\rangle$, measuring it on $\hat{A}$ or $\hat{B}$ would not give a certain result because $\hat{A}$ and $\hat{B}$ do not commute with $\hat{H}_{\mathrm{q}}$. Thus, one would not be able to distinguish the exact eigenstate from other states by its zero variance. The origin of the discrepancy between quantum uncertainty given by the variance (Var) of $\hat{H}_{\mathrm{q}}$ (true uncertainty) and by the sum of variances for $\hat{A}$ and $\hat{B}$ is neglect of covariances (Cov)

$$
\begin{gathered}
\operatorname{Var}\left(\hat{H}_{\mathrm{q}}\right)=\operatorname{Var}(\hat{A})+\operatorname{Var}(\hat{B})+\operatorname{Cov}(\hat{A}, \hat{B})+\operatorname{Cov}(\hat{B}, \hat{A}), \\
\operatorname{Var}(\hat{A})=\left\langle\hat{A}^{2}\right\rangle-\langle\hat{A}\rangle^{2}, \\
\operatorname{Cov}(\hat{A}, \hat{B})=\langle\hat{A} \hat{B}\rangle-\langle\hat{A}\rangle\langle\hat{B}\rangle .
\end{gathered}
$$


Thus, even though the $\hat{H}_{\mathrm{q}}$ average is equal to averages of $\hat{A}$ and $\hat{B}$, the true quantum uncertainty of $\hat{H}_{\mathrm{q}}$ is overestimated by a sum of variances for $\hat{A}$ and $\hat{B}$. Moreover, the number of measurements to sample $\hat{A}$ and $\hat{B}$ is twice as many as that for $\hat{H}_{\mathrm{q}}$ if the eigenstate nature of $U\left|\Psi_{0}\right\rangle$ is not known a priori.

The variance of any Hamiltonian depends only on the Hamiltonian and the wavefunction, but if one approximates the variance using only variances of Hamiltonian parts and neglects covariances between the parts, the result of such an approximation will depend on the partitioning. Importantly, the sum of variances for the Hamiltonian parts can either under- or overestimate the true Hamiltonian variance. To see how ignoring covariances can erroneously make estimates of the uncertainty arbitrarily small consider an artificial example, where the Hamiltonian variance is measured as $n$ independent measurements of its $\hat{H}_{\mathrm{q}} / n$ identical parts. Due to the linear scaling of the variance sum with $n$ and the inverse quadratic scaling of variances of individual terms with $n$, the overall scaling of the variance is inversely proportional to $n$ and can be made arbitrarily small by choosing large enough $n$. This follows from a wrong assumption that parts $\left(\hat{H}_{\mathrm{q}} / n\right)$ are independent and covariances between them are zero.

Generally, the number of non-commuting terms in $\hat{H}_{\mathrm{q}}$ grows with the size of the original molecular problem, and the total uncertainty from the measurement of individual terms will increase. This increase raises the standard deviation of the total measurement process and leads to a large number of measurements to reach convergence in the energy expectation value. The question we would like to address is whether it is possible to reduce the number of the $\hat{H}_{\mathrm{q}}$ terms that needs to be measured separately.

In this paper we introduce a new systematic approach to decreasing uncertainty of the expectation energy measurement. We substitute the conventional measurement partitioning of the Hamiltonian with groups of qubit-wise commuting operators ${ }^{13,14}$ by partitioning to terms whose eigenstates can be found exactly using the mean-field procedure. Owing to a more general structure of such terms the Hamiltonian can be split into a fewer number of them. Interestingly, the general operator conditions on such mean-field terms have not been found in the literature and have been derived in this work for the first time. To decrease the number of these terms even further, we augment the mean-field treatment with few-qubit unitary transformations that allow us to measure few-qubit entangled terms. Measurement of newly introduced terms requires the scheme appearing in the cluster-state quantum computing, ${ }^{15,16}$ it is qubit-wise measurement with use of previous measurement results to define what single-qubit operators to measure next.

\section{Theory}

\subsection{Qubit Hamiltonian}

In order to formulate the electronic structure problem for a quantum computer that operates with qubits (two-level systems), the electronic Hamiltonian needs to be transformed iso-spectrally to its qubit form. This is done in two steps. First, the second quantized form of $\hat{H}_{\mathrm{e}}$ is obtained

$$
\hat{H}_{\mathrm{e}}=\sum_{p q} h_{p q} \hat{a}_{p}^{\dagger} \hat{a}_{q}+\frac{1}{2} \sum_{p q r s} g_{p q r s} \hat{a}_{p}^{\dagger} \hat{a}_{q}^{\dagger} \hat{a}_{s} \hat{a}_{r}
$$

where $\hat{a}_{p}^{\dagger}\left(\hat{a}_{p}\right)$ are fermionic creation (annihilation) operators, $h_{p q}$ and $g_{p q r s}$ are one- and two-electron integrals in a spin-orbital basis. ${ }^{17}$ This step has polynomial complexity and is carried out on a classical computer. Then, using the $\mathrm{JW}^{6,7}$ or more resourceefficient BK transformation, ${ }^{8-12}$ the electronic Hamiltonian is converted iso-spectrally to a qubit form

$$
\hat{H}_{\mathrm{q}}=\sum_{I} C_{I} \hat{P}_{I},
$$

where $C_{I}$ are numerical coefficients, and $\hat{P}_{I}$ are Pauli "words", products of Pauli operators of different qubits

$$
\hat{P}_{I}=\cdots{\hat{\sigma_{2}}}^{(I)}{\hat{\sigma_{1}}}^{(I)},
$$

$\hat{\sigma}_{i}^{(I)}$ is one of the $\hat{x}, \hat{y}, \hat{z}$ Pauli operators for the $i^{\text {th }}$ qubit. The number of qubits $N$ is equal to the number of spin-orbitals used in the second quantized form [eqn (4)]. Since every fermionic operator is substituted by a product of Pauli operators in both JW and BK transformations, the total number of Pauli words in $\hat{H}_{\mathrm{q}}$ scales as $N^{4}$.

\subsection{Conventional measurement}

In the conventional VQE scheme the $\hat{H}_{\mathrm{q}}$ is separated into sums of qubit-wise commuting (QWC) terms,

$$
\begin{gathered}
\hat{H}_{\mathrm{q}}=\sum_{n} \hat{A}_{n},\left[\hat{A}_{n}, \hat{A}_{k}\right]_{\mathrm{qw}} \neq 0, \quad \text { if } n \neq k \\
\hat{A}_{n}=\sum_{I} C_{I}{ }^{(n)} \hat{P}_{I}^{(n)},\left[\hat{P}_{I}^{(n)}, \hat{P}_{J}^{(n)}\right]_{\mathrm{qw}}=0, \quad \forall I \& J .
\end{gathered}
$$

Here $\left[\hat{P}_{I}^{(n)}, \hat{P}_{J}^{(n)}\right]_{\mathrm{qw}}$ denotes a qubit-wise commutator of two Pauli words, it is zero only if all one-qubit operators in $\hat{P}_{I}^{(n)}$ commute with their counterparts in $\hat{P}_{J}^{(n)}$. Clearly, if $\left[\hat{P}_{I}^{(n)}, \hat{P}_{J}^{(n)}\right]_{\mathrm{qw}}$ then the normal commutator is $\left[\hat{P}_{I}^{(n)}, \hat{P}_{J}^{(n)}\right]=0$. The opposite is not true, a simple example is $\left[\hat{x}_{1} \hat{x}_{2}, \hat{y}_{1} \hat{y}_{2}\right]=0$ but $\left[\hat{x}_{1} \hat{x}_{2}, \hat{y}_{1} \hat{y}_{2}\right]_{\mathrm{qw}} \neq$ 0 . We will not be using non-zero results of the qubit-wise commutator and therefore their exact values are not important, but it is assumed that $[.,]_{\mathrm{qw}}$ is bi-linear for both operators.

Partitioning of the $H_{\mathrm{q}}$ in eqn (7) allows one to measure all Pauli words within each $\hat{A}_{n}$ term in a single set of $N$ one-qubit measurements. For every qubit, it is known from the form of $\hat{A}_{n}$, what Pauli operator needs to be measured. The advantage of this scheme is that it requires only single-qubit measurements, which are technically easier than multi-qubit measurements. The disadvantage of this scheme is that the Hamiltonian may require measuring too many $\hat{A}_{n}$ terms separately.

A natural extension of partitioning in eqn (7) is to sum more general terms

$$
\hat{H}_{\mathrm{q}}=\sum_{n} \hat{H}_{n}^{(\mathrm{MF})}
$$

with the condition that $\hat{H}_{n}^{(\mathrm{MF})}$ eigenstates can be presented in a single-product form of single-qubit wavefunctions. In other words, the eigenstates of the $\hat{H}_{n}^{(\mathrm{MF})}$ fragments are unentangled 
and can be obtained using a mean-field procedure. This condition would allow measurement of each $\hat{H}_{n}^{(\mathrm{MF})}$ fragment qubit after a qubit. However, to perform the new splitting we need an exact definition of the mean-field (MF) Hamiltonian so that we can recognize these new blocks within the total Hamiltonian.

\subsection{Mean-field Hamiltonians}

What is the most general form of a qubit Hamiltonian whose eigenstates can be presented as single factorized products of one-qubit wavefunctions? Note that the well-known example of such Hamiltonians, separable operators,

$$
\hat{H}_{\mathrm{S}}(1, \ldots, N)=\sum_{i=1}^{N} \hat{h}_{i}(i)
$$

are a particular class that does not provide the most general form. In other words, there are many more Hamiltonians that are not separable but are still in the MF class, one simple example is

$$
\hat{H}_{\mathrm{MF}}(1,2)=\hat{x}_{2}+\hat{z}_{1} \hat{y}_{2},
$$

which does not follow the form of eqn (10) but whose eigenstates, $\left|+_{z}\right\rangle_{1}\left| \pm_{x+y}\right\rangle_{2}$ and $|-z\rangle_{1}\left| \pm_{x-y}\right\rangle_{2}, \ddagger$ are unentangled products.

We formulate the general criterion for a Hamiltonian $H(1, \ldots N)$ to be in the MF class as follows. There should exist $N$ one-particle operators $\left\{\hat{O}_{k}(k)\right\}_{k=1}^{N} \S$ that commute $\left[\hat{O}_{k}, \hat{H}_{N-k+1}\right]=$ 0 with the system of $N$ Hamiltonians $\left\{\hat{H}_{N-k+1}\right\}_{k=1}^{N}$ constructed in the following way that we will refer as a reductive chain:

$$
\begin{aligned}
& 1: \hat{H}_{N}=\hat{H}, \\
& 2: \hat{H}_{N-1}=\left\langle\phi_{1}\left|\hat{H}_{N}\right| \phi_{1}\right\rangle, \\
& \cdots \\
& N: \hat{H}_{1}=\left\langle\phi_{N-1}\left|\hat{H}_{2}\right| \phi_{N-1}\right\rangle,
\end{aligned}
$$

where $\hat{O}_{k}\left|\phi_{k}\right\rangle=\lambda_{k}\left|\phi_{k}\right\rangle$. The final operator in this chain is a oneparticle operator that commutes with itself and defines $\hat{O}_{N}=$ $\hat{H}_{1}$. The proof of this criterion can be found in Appendix A. It is easy to see that $|\Psi\rangle=\prod_{k=1}^{N}\left|\phi_{k}\right\rangle$ is an eigenfunction of $\hat{H}$. Clearly, separable Hamiltonians are in the MF class because for them, $\hat{O}_{k}$ 's can be taken as $\hat{h}_{k}(k)$ from eqn (10). However, note that because the system of $\hat{O}_{k}$ operators is required to commute not with $\hat{H}$ but with the reduced set of Hamiltonians, the formulated criterion goes beyond separable Hamiltonians.

A general procedure to determine whether a particular qubit Hamiltonian $\hat{H}$ is in the MF class or not requires finding all $N$ one-particle operators $\hat{O}_{k}$. The procedure starts with a check whether there is at least one qubit $k$ for which

$$
\left[\hat{H},\left(a \hat{x}_{k}+b \hat{y}_{k}+c \hat{z}_{k}\right)\right]=0
$$

can be achieved by choosing a non-zero vector $(a, b, c)$. Once the first operator $\hat{O}_{1}(k)=a \hat{x}_{k}+b \hat{y}_{k}+c \hat{z}_{k}$ is found its eigenstates can be integrated out to generate $\hat{H}_{N-1}$, and the procedure can be repeated to find $\hat{O}_{2}$ that commutes with $\hat{H}_{N-1}$.

\subsection{Measurement of mean-field Hamiltonians}

Measuring an $N$-qubit mean-field Hamiltonian can be done by performing a single set of sequential $N$ one-qubit measurements. Each qubit projective measurement in this set will collapse the measured wavefunction to an eigenstate of the corresponding single qubit operator. The single qubit operators that need to be measured are $\hat{O}_{k}$ 's operators. The definition of one particle operators may depend on the result of the previous measurement. Let us consider the mean-field Hamiltonian in eqn (11): $\hat{O}_{1}(1)=\hat{z}_{1}$, and $\hat{O}_{2}(2)=\hat{x}_{2} \pm \hat{y}_{2}$, where \pm is determined by the eigenfunction chosen from the $\hat{O}_{1}$ spectrum to generate the $\hat{H}_{1}=\left\langle\phi_{1}{ }^{ \pm}\left|\hat{H}_{\mathrm{MF}}\right| \phi_{1}{ }^{ \pm}\right\rangle$in the chain of eqn (12). This ambiguity does not allow one to present $\hat{H}_{\mathrm{MF}}$ as an operator with all qubitwise commuting components. An attempt on this can be done by inserting the projectors on the eigenstates of $\hat{z}_{1}$ instead of the operator:

$$
\begin{aligned}
\hat{H}_{\mathrm{MF}} & =\left(\hat{x}_{2}+\hat{y}_{2}\right)\left|\phi_{1}{ }^{+}\right\rangle\left\langle\phi_{1}{ }^{+}\left|+\left(\hat{x}_{2}-\hat{y}_{2}\right)\right| \phi_{1}{ }^{-}\right\rangle\left\langle\phi_{1}{ }^{-}\right| \\
\hat{H}_{\mathrm{MF}} & =\left[\left(\hat{x}_{2}+\hat{y}_{2}\right)\left(1+\hat{z}_{1}\right)+\left(\hat{x}_{2}-\hat{y}_{2}\right)\left(1-\hat{z}_{1}\right)\right] / 2,
\end{aligned}
$$

where $\hat{z}_{1}\left|\phi_{1}{ }^{ \pm}\right\rangle= \pm\left|\phi_{1}{ }^{ \pm}\right\rangle$, and even though the projectors onto the $\left|\phi_{1}{ }^{ \pm}\right\rangle$eigenstates commute, the $\left(\hat{x}_{2} \pm \hat{y}_{2}\right)$ parts do not.

Therefore, the scheme for measuring the $\hat{H}_{\mathrm{MF}}$ will be as shown in Fig. 1. Note that no matter how entangled the initial wavefunction is, measuring $\hat{H}_{\mathrm{MF}}$ does not require measuring $\hat{x}_{2}$ and $\hat{z}_{1} \hat{y}_{2}$ separately as was done in the regular VQE scheme.

In practice, qubit-wise measurements using previous measurement results to define what single-qubit operators to measure next, or feedforward measurements, have been implemented in quantum computers based on superconductor and photonic qubit architectures. ${ }^{18,19}$ The essential feasibility condition for the feedforward measurement is that the delay introduced by measurements is much shorter than the qubit coherence time. For superconducting (photonics) qubit architectures this condition has been achieved with typical timescales for a measurement and coherence as $2 \mu \mathrm{s}$ (ref. 20) (150 ns (ref. 19)) and $40 \mu$ s (ref. 21) (100 ms (ref. 22)), respectively.

\subsection{Mean-field partitioning}

Even though regular molecular qubit Hamiltonians are not guaranteed to be in the MF class, it is always possible to split any $N$-qubit Hamiltonian into a sum of MF Hamiltonians. To see this, we will present a heuristic partitioning scheme that guarantees the MF partitioning.

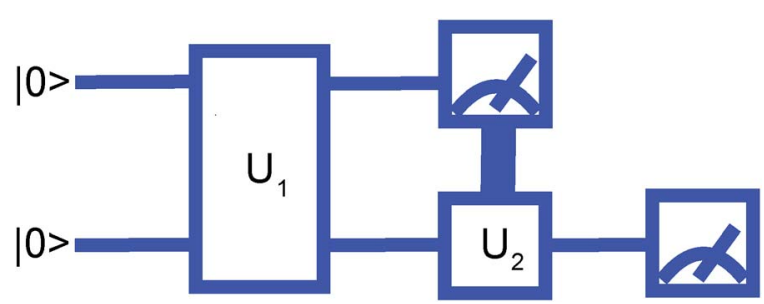

Fig. 1 Measurement where the second qubit is rotated by $U_{2}$ depending on the result of the first qubit measurement. 
Our scheme uses ranking of all qubits $k=1, \ldots, N$ based on a geometrical characteristic $l(k)$, which is defined as follows. For an arbitrary qubit $k$, the total Hamiltonian can be written as

$$
\hat{H}=\hat{h}_{x} \hat{x}_{k}+\hat{h}_{y} \hat{y}_{k}+\hat{h}_{z} \hat{z}_{k}+\hat{h}_{e}
$$

where $\hat{h}_{x, y, z, e}$ are the residual operators that do not contain Pauli matrices for the $k^{\text {th }}$ qubit. Assembling coefficients of Pauli words in operators $\hat{h}_{x, y, z}$ into vectors, $\bar{h}_{x, y, z}$, we build matrix $A_{k}=$ $\left[\bar{h}_{x} \bar{h}_{y} \bar{h}_{z}\right]$ with dimensions $M$ by 3 , where $M$ is the number of different Pauli words in $\hat{h}_{x, y, z}$ operators. To define $l(k)$, we evaluate matrix $S_{k}=A_{k}^{*} A_{k}$ and assign $l(k)=\operatorname{dim}\left(\operatorname{ker}\left(S_{k}\right)\right)$. Evaluating $S_{k}$ is equivalent to obtaining the overlap between three vectors $\bar{h}_{x, y, z}$ assuming the orthogonal basis, while the dimensionality of its kernel is the number of its zero eigenvalues.

$l(k)$ allows one to answer a question on whether there is a transformation involving only the $k^{\text {th }}$ qubit that can present $\hat{H}$ in one of the two forms:

$$
\begin{gathered}
\hat{H}=\hat{h} \hat{O}_{k}+\hat{h}_{e}, \\
\hat{H}=\hat{h}^{\prime} \hat{O}_{k}^{\prime}+\hat{h}^{\prime \prime} \hat{O}_{k}^{\prime \prime}+\hat{h}_{e},
\end{gathered}
$$

where $\hat{O}_{k}, \hat{O}_{k}^{\prime}, \hat{O}_{k}^{\prime \prime}$ are operators containing only the $k^{\text {th }}$ qubit, and $\hat{h}, \hat{h}^{\prime}, \hat{h}^{\prime \prime}$ are the complementary operators that exclude the $k^{\text {th }}$ qubit. The positive answers in the forms of eqn (17) and (18) correspond to $l(k)=2$ and $l(k)=1$, respectively. $l(k)=2$ is equivalent to the MF condition of eqn (13), with $\hat{O}_{k}=a \hat{x}_{k}+b \hat{y}_{k}+$ $c \hat{z}_{k}$. For $l(k)=1$, the MF treatment of the $k^{\text {th }}$ qubit is not possible but using eqn (18) the $k^{\text {th }}$ qubit dependence in the Hamiltonian can be somewhat compactified. Coefficients for $\hat{O}_{k}, \hat{O}_{k}^{\prime}, \hat{O}_{k}^{\prime \prime}$ and $\hat{h}, \hat{h}^{\prime}, \hat{h}^{\prime \prime}$ operators can be found from non-zero eigenvectors of $S_{k}$ (this process is detailed in Appendix B). The negative answer to the question leaves $\hat{H}$ in the original form of eqn (16) and is equivalent to $l(k)=0$.

The question about possible compactification of the $k^{\text {th }}$ qubit dependence in the Hamiltonian has a simple geometric interpretation in terms of arrangement of the three vectors $\bar{h}_{x, y, z}$. These multi-dimensional vectors can be linearly independent (eqn (16)), located within some plane (eqn (18)), or collinear to each other (eqn (17)), Fig. 2 illustrates all three cases.

Using a set of $l(k)$ 's for a given Hamiltonian one can decide how many qubits can be treated using the MF procedure, these will be all qubits with $l(k)=2$. Once all of such qubits have been considered, the MF partitioning of $l(k)=1$ qubits begins. For $l(k)=1$, the Hamiltonian can be split for any of such qubits into two parts: $\hat{H}^{(1)}=\hat{h}^{\prime} \hat{O}_{k}^{\prime}$ and $\hat{H}^{(2)}=\hat{h}^{\prime \prime} \hat{O}_{k}^{\prime \prime}+\hat{h}_{e}$. In both parts the $k^{\text {th }}$ qubit can be treated using the MF treatment, which allows one to continue the consideration for $\hat{h}^{\prime}, \hat{h}^{\prime \prime}$ and $\hat{h}_{e}$. Finally, if only qubits with $l(k)=0$ are left, then $\hat{H}$ needs to be partitioned to three Hamiltonians $\hat{H}^{(1)}=\hat{h}_{x} \hat{x}_{k}, \hat{H}^{(2)}=\hat{h}_{y} \hat{y}_{k}$, and $\hat{H}^{(3)}=\hat{h}_{z} \hat{z}_{k}+$ $\hat{h}_{e}$, where at least the $k^{\text {th }}$ qubit can be treated using MF. After this separation one can apply the reduction chain to each of the three operators. Fig. 3 illustrates the partitioning for a three qubit case detailed in Appendix B. In the case when reducing the $k^{\text {th }}$ qubit does not produce a Hamiltonian with reducible qubits the partitioning needs to be repeated, as in Fig. 3 when
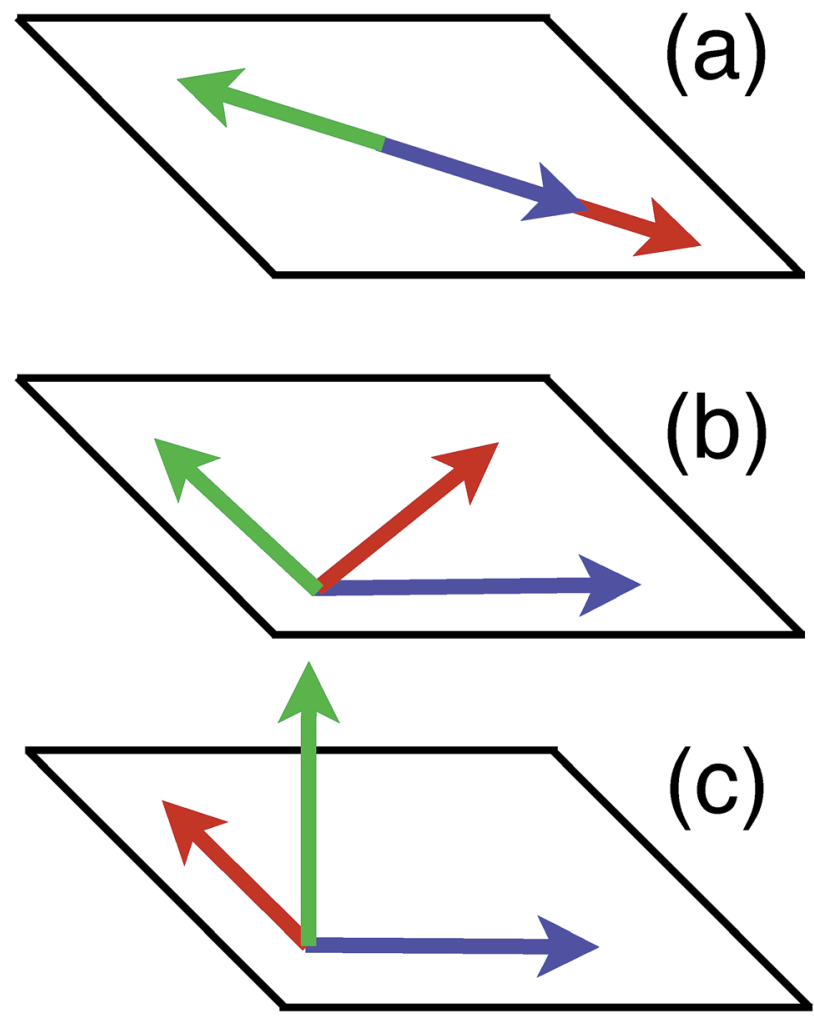

Fig. 2 Possible geometrical arrangement of three multi-dimensional vectors $h_{x, y, z}$ (green, blue, and red arrows): (a) collinear arrangement $[l(k)=2]$, (b) planar arrangement $[l(k)=1]$, (c) linearly independent case $[l(k)=0]$

transforming qubit 1 led to $h(2,3)$ where none of the qubits can be reduced.

Our scheme can be considered as an example of a greedy algorithm because at every step it tries to find locally the most optimal reduction, a qubit with the highest $l(k)$. The reduction is only possible if there is linear dependency between complementary vectors $\vec{h}_{x, y, z}$. The lower the dimensionality of the linear space, where these vectors are located, the more probable such linear dependence. Thus, treating qubits with the highest $l(k)$ first is justified by the reduction of the space dimensionality along the reductive scheme. In the example of Fig. 3 treatment of qubits 2 and 3 in the beginning would require partitioning of the Hamiltonian to two branches for each of them, while leaving the $3^{\text {rd }}$ qubit to the end did not generate any new terms for it.

It is possible that more than one qubit will have the highest $l(k)$. To do more optimal selection in this case, one would need to consider maxima of $l(k)$ functions on qubits that enter complementary Hamiltonians $\hat{h}$ for different reduction candidates. This consideration makes the partitioning computationally costly and was not performed in this work.

Applying the partitioning scheme guarantees to result in a sum of MF Hamiltonians that can be measured in $\mathrm{N}$-qubit one-particle measurements. Since any linear combination of QWC terms form a MF Hamiltonian, this partitioning scheme 


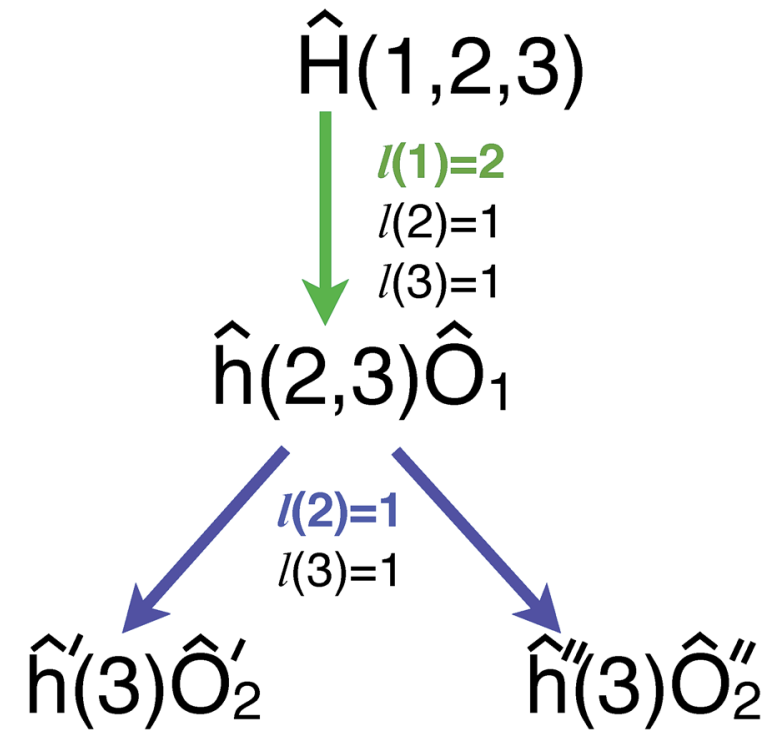

Fig. 3 The MF partitioning scheme uses the $l(k)$ function at each step to split a three qubit Hamiltonian (detailed in Appendix B) into two fragments. The MF partitioned form is $\hat{H}(1,2,3)=\hat{h}^{\prime}(3) \hat{O}_{2}^{\prime} \hat{O}_{1}+\hat{h}^{\prime \prime}(3) \hat{O}_{2}^{\prime \prime} \hat{O}_{1}$, where all qubits in both fragments can be treated using the MF procedure.

cannot produce more terms than those used in the regular VQE measuring scheme.

\subsection{Unitary transformations generating mean-fields}

Partitioning the non-MF blocks in the Hamiltonian to obtain more MF terms leads to growth of the terms needed to be measured. An alternative treatment of non-MF groups is to search for multi-qubit operators that commute with them. Finding such operators may lead to unitary transformations that can transform non-MF Hamiltonians into Hamiltonians where qubits shared with the commuting operator can be treated using the mean-field procedure. Similar search for multi-qubit operators commuting with the system Hamiltonian was used recently by Bravyi and coworkers to reduce the qubit count in the conventional VQE scheme. ${ }^{23}$

Let us consider an example where an $\mathrm{N}$-qubit non-MF Hamiltonian $\hat{H}$ has a two-qubit operator $\hat{O}^{(2)}(1,2)$ commuting with it (without loss of generality we can assume that $\hat{O}^{(2)}$ acts on the first two qubits). Then, under certain conditions detailed in Appendix A, $\hat{H}$ allows for its eigenstates $\Psi$ to be written as $\Psi(1, \ldots N)=\Phi(1,2) \psi(3, \ldots N)$, where $\Phi(1,2)$ is an eigenstate of $\hat{O}^{(2)}$. One can always write $\Phi(1,2)=\hat{U}(1,2) \phi_{1}(1) \phi_{2}(2)$, where $\hat{U}(1,2)$ is an operator entangling the product state $\phi_{1}(1) \phi_{2}(2)$ into $\Phi(1,2)$. Using this unitary operator, one can obtain the Hamiltonian $\hat{H}_{12}=\hat{U}(1,2)^{\dagger} \hat{H} \hat{U}(1,2)$ that has an eigenstate $\Psi_{12}(1, \ldots N)=\phi_{1}(1)$ $\phi_{2}(2) \psi(3, \ldots N)$ where qubits 1 and 2 are unentangled. Therefore, there should be one-particle operators of qubits 1 and 2 that commute with $\hat{H}_{12}$ and its MF-reduced counterpart. Finding these operators and their eigenfunctions $\phi_{1}(1)$ and $\phi_{2}(2)$ allows us to integrate out qubits 1 and 2

$$
\hat{H}_{N-2}=\left\langle\phi_{1} \phi_{2}\left|H_{12}\right| \phi_{1} \phi_{2}\right\rangle .
$$

Search for one- or multi-qubit operators commuting with $\hat{H}_{N-2}$ can be continued. The procedure to find commuting operators with increasing number of qubits requires exponentially increasing number of variables parametrizing such operators. Indeed, a $k$-qubit operator requires a $3^{k}$ coefficient for all Pauli words in commutation equations similar to eqn (13), also the number of different $k$-qubit operators among $N$ qubits is $C_{N}{ }^{k}$ $\sim N_{k}$. Potentially, such operators always exist (e.g., projectors on eigenstates of the Hamiltonian) but the amount of resources needed for their search can exceed what is available. Thus we recommend interchanging this search with the partitioning described above if the multi-qubit search requires going beyond 2-qubit operators.

To illustrate the complete scheme involving multi-qubit transformations, let us assume that we can continue the reduction chain for $\hat{H}=\hat{H}_{N}$ by generating the set of Hamiltonians $\left\{\hat{H}_{N}, \hat{H}_{N-2}, \ldots, \hat{H}_{k}\right\}$ using qubit unitary transformations $\{U(1,2), U(3,4,5), \ldots, U(N-k, \ldots N)\}$ and integrating out variables from $N$ to $k$. To take advantage of this reduction chain in measuring an expectation value of an arbitrary wavefunction $\chi(1, \ldots N)$ on $\hat{H}$, such a measurement should be substituted by the following set of conditional measurements:

Step 1: first two qubits are measured using $\hat{H}_{12}$ and the unitary transformed function $\left|\hat{U}(1,2)^{\dagger} \chi\right\rangle$ because

$$
\begin{aligned}
\langle\chi|\hat{H}| \chi\rangle & =\left\langle\chi\left|\hat{U}(1,2) \hat{H}_{12} \hat{U}(1,2)^{\dagger}\right| \chi\right\rangle \\
& =\left\langle\hat{U}(1,2)^{\dagger} \chi\left|\hat{H}_{12}\right| \hat{U}(1,2)^{\dagger} \chi\right\rangle .
\end{aligned}
$$

Depending on the results of these measurements the operator $\hat{H}_{N-2}$ is formulated and its unitary transformation $U(3,4,5)$ is found. $U(3,4,5)$ gives rise to the transformed Hamiltonian $\hat{H}_{35}=\hat{U}(3,4,5)^{\dagger} \hat{H}_{N-2} \hat{U}(3,4,5)$. The wavefunction after measuring qubits 1 and 2 is denoted as $\left|\chi_{12}\right\rangle$.

Step 2: qubits 3-5 are measured on $\hat{H}_{35}$ sequentially using the transformed wavefunction $\hat{U}(3,4,5)^{\dagger}\left|\chi_{12}\right\rangle$. Results of these measurements will define the next reduction step and the wavefunction that should be unitarily transformed for the next measurement.

These steps can be continued until all qubits have been measured. If resources allow for finding corresponding multiqubit unitary transformations, the $\hat{H}$ Hamiltonian can be measured in $N$ single-qubit measurements.

\section{Numerical studies and discussion}

To assess our developments we apply them to the Hamiltonians of the $\mathrm{H}_{2}$ and LiH molecules obtained within the STO-3G basis and used to illustrate the performance of quantum computing techniques previously. ${ }^{13,24,25}$

\section{$3.1 \quad \mathrm{H}_{2}$ molecule}

The BK-transformed qubit Hamiltonian contains the following terms: 


$$
\begin{aligned}
\hat{H}_{\mathrm{H}_{2}}= & C_{0}+C_{1} \hat{z}_{2}+C_{2} \hat{z}_{3}+C_{3} \hat{z}_{4}+C_{4} \hat{z}_{1} \hat{z}_{3}+C_{5} \hat{z}_{2} \hat{z}_{4} \\
& +C_{6} \hat{z}_{3} \hat{z}_{4}+C_{7} \hat{z}_{1} \hat{z}_{2} \hat{z}_{3}+C_{8}\left(1+\hat{z}_{1}\right) \hat{z}_{2} \hat{z}_{3} \hat{z}_{4}+C_{9} \hat{z}_{1} \hat{z}_{2} \hat{z}_{4} \\
& +C_{10}\left(1+\hat{z}_{1}\right) \hat{y}_{2} \hat{z}_{3} \hat{z}_{4}+C_{11}\left(1+\hat{z}_{1}\right) \hat{x}_{2} \hat{z}_{3} \hat{x}_{4} .
\end{aligned}
$$

where some of the $C_{i}$ 's are equal, but it is not going to be important for us (the details of generating this Hamiltonian are given in Appendix C). Clearly $\hat{H}_{\mathrm{H}_{2}}$ contains three groups of QWC terms, the first three lines form one group, and the two last terms fall into two other groups. $\hat{H}_{\mathrm{H}_{2}}$ is not a MF Hamiltonian, only qubits 1 and 3 have one-particle operators commuting with the Hamiltonian, while after their reduction the reduced Hamiltonian does not commute with any one-particle operator

$$
\hat{H}_{24}=D_{0}+D_{1} \hat{z}_{2}+D_{2} \hat{z}_{4}+D_{3} \hat{z}_{2} \hat{z}_{4}+D_{4} \hat{x}_{2} \hat{x}_{4}+D_{5} \hat{y}_{2} \hat{y}_{4},
$$

where $D_{i}$ 's are constants. Partitioning of $\hat{H}_{24}$ to three terms using qubit 2 or 4 would not be more efficient than partitioning $\hat{H}_{\mathrm{H}_{2}}$ in 3 groups of QWC terms from the beginning. However, there is the two-particle operator $\hat{z}_{2} \hat{\mathrm{z}}_{4}$ that commutes with $\hat{H}_{24}$, and it can be used to devise a unitary transformation bringing $\hat{H}_{24}$ to the MF form. Note that even though $\hat{z}_{2} \hat{z}_{4}$ has a spectral degeneracy, this degeneracy will not create problematic entanglement discussed in Appendix A, because there are no other qubits besides 2 and 4 in $\hat{H}_{24}$. The sought unitary transformation is $U(2,4)=\exp \left[-\mathrm{i}(3 \pi / 2) \hat{z}_{2} \hat{x}_{4}\right]$, and the transformed MF Hamiltonian is

$$
\begin{aligned}
U(2,4)^{\dagger} \hat{H}_{24} U(2,4)= & E_{0}+E_{1} \hat{z}_{2}+E_{2} \hat{y}_{2}+E_{3} \hat{y}_{4} \\
& +E_{4} \hat{y}_{2} \hat{y}_{4}+E_{5} \hat{z}_{2} \hat{y}_{4},
\end{aligned}
$$

where $E_{i}$ 's are some constants and the first one-particle commuting operator is $\hat{O}_{1}^{(4)}=\hat{y}_{4}$. After integrating out $\hat{O}_{1}$ 's eigenfunction, $\hat{O}_{2}{ }^{(2)}$ is a linear combination of $\hat{z}_{2}$ and $\hat{y}_{2}$.

To illustrate the superiority of the scheme with the use of $U(2,4)$ and measurements of the MF Hamiltonian over the regular approach with splitting $\hat{H}_{\mathrm{H}_{2}}$ to three groups of QWC operators, Table 1 presents variances for the Hamiltonian expectation value for two wavefunctions, the exact eigenfunction $\left(\Psi_{\mathrm{QCC}}\right)$ of and the mean-field approximation $\left(\Psi_{\mathrm{QMF}}\right)$ to the ground state of the $\mathrm{H}_{2}$ problem at $R(\mathrm{H}-\mathrm{H})=1.5 \AA .{ }^{25}$ The exact solution measured in the new scheme (MF-partitioning 2p) gives only one value with zero variance, while the regular schemes give three distributions for each non-commuting term.

In the approximate wavefunction case, the true variance obtained from the Hamiltonian is larger than that of the conventional approach. This is a consequence of ignoring covariances in the conventional approach. The MF partitioning $2 p$ variance is equal to the exact one, since it is obtained from measuring a single term (the MF Hamiltonian in eqn (23)) and thus does not neglect any covariances.

\section{2 $\quad$ LiH molecule}

We will consider the $\mathrm{LiH}$ molecule at $R(\mathrm{Li}-\mathrm{H})=3.2 \AA$, it has a 6qubit Hamiltonian containing 118 Pauli words (see Appendix C for details). This qubit Hamiltonian has $3^{\text {rd }}$ and $6^{\text {th }}$ stationary qubits, which allow one to replace the corresponding $\hat{z}$ operators by their eigenvalues, \pm 1 , thus defining the different
Table 1 Estimates of total variances (Var) for the $\mathrm{H}_{2}$ and $\mathrm{LiH}$ molecules with different partitioning approaches and wavefunctions ( $\Psi_{\mathrm{QCC}}$ from the qubit coupled cluster method, ${ }^{25}$ and $\Psi_{\mathrm{QMF}}$ from the qubit meanfield approach $\left.{ }^{26}\right)^{a}$

\begin{tabular}{llll}
\hline Approach & Number of terms & $\operatorname{Var}\left(\Psi_{\mathrm{QCC}}\right)$ & $\operatorname{Var}(\Psi$ \\
\hline $\mathbf{H}_{\mathbf{2}}$ & & & \\
QWC-partitioning & 3 & 0.044 & 0.026 \\
MF-partitioning 2p & 1 & 0 & 0.053 \\
$\left\langle\hat{H}_{\mathrm{H}_{2}}{ }^{2}\right\rangle-\left\langle\hat{H}_{\mathrm{H}_{2}}\right\rangle^{2}$ & 1 & 0 & 0.053 \\
LiH & & & \\
QWC-partitioning & 25 & 0.043 & 0.037 \\
MF-partitioning 1p & 13 & 0.029 & 0.036 \\
MF-partitioning 2p & 5 & 0.030 & 0.038 \\
$\left\langle\hat{H}_{\mathrm{LiH}}{ }^{2}\right\rangle-\left\langle\hat{H}_{\mathrm{LiH}}\right\rangle^{2}$ & 1 & $5.6 \times 10^{-4}$ & 0.027
\end{tabular}

${ }^{a}$ The number of terms corresponds to the number of separately measured $\mathrm{N}$-qubit terms. For all partitionings, covariances have not been included in the Var estimates, which simulates practical estimation of the total variance.

"sectors" of the original Hamiltonian. Each of these sectors is characterized by its own 4-qubit effective Hamiltonian. The ground state lies in the $z_{3}=-1, z_{6}=1$ sector; the corresponding 4-qubit effective Hamiltonian $\left(\hat{H}_{\mathrm{LiH}}\right)$ has 100 Pauli terms. Integrating out $3^{\text {rd }}$ and $6^{\text {th }}$ qubits can be done in the MF framework. The MF treatment of $\hat{H}_{\mathrm{LiH}}$ is not possible without its partitioning.

Before discussing partitioning of $\hat{H}_{\mathrm{LiH}}$ it is worth noting that there are two 2-qubit operators commuting with $\hat{H}^{(4)}$ (we reenumerate qubits after the reduction from 6 to 4 qubits in the Hamiltonian)

$$
\begin{aligned}
& \hat{O}_{1}{ }^{(2)}=-\hat{z}_{1}+\hat{z}_{2}-\hat{z}_{1} \hat{z}_{2} \\
& \hat{O}_{2}{ }^{(2)}=-\hat{z}_{3}+\hat{z}_{4}+\hat{z}_{3} \hat{z}_{4} .
\end{aligned}
$$

Unfortunately, both operators have degenerate spectra with a single non-degenerate eigenstate and three degenerate states. Moreover, these degeneracies do not satisfy the factorability condition introduced in Appendix A thus proving it impossible to find 2-qubit unitary transformation that would factorize qubits 1 and 2 or 3 and 4 .

Table 1 summarizes results of partitioning for $\hat{H}_{\mathrm{LiH}}$ and variances calculated for different wavefunctions and partitioning schemes. The partitioning involving only one-qubit transformations (MF-partitioning 1p) reduces the number of QWC terms by half. Involving the two-qubit transformations at the

Table 2 Commutativity of two operators and their simultaneous single-qubit measurability (SQM)

\begin{tabular}{llll}
\hline$\hat{A}$ & $\hat{B}$ & {$[\hat{A}, \hat{B}]$} & SQM of $(\hat{A}+\hat{B})$ \\
\hline$\hat{z}_{1} \hat{z}_{2}$ & $\hat{z}_{2} \hat{z}_{3}$ & 0 & Yes \\
$\hat{z}_{1} \hat{z}_{2}$ & $\hat{x}_{1} \hat{x}_{2}$ & 0 & No \\
$\hat{z}_{1} \hat{z}_{3}$ & $\hat{x}_{1} \hat{z}_{2}$ & $\neq 0$ & Yes \\
$\hat{z}_{1} \hat{z}_{2}$ & $\hat{x}_{1} \hat{y}_{2}$ & $\neq 0$ & No
\end{tabular}


step before the last one in the MF partitioning reduces the number of terms to only 5 (MF-partitioning 2p), which is a fivefold reduction compared to the conventional QWC form. Alternative pathways in the MP partitioning scheme related to different choices of partitioned qubits with the same value of $l(k)$ generated not more than 15 and 9 terms for MF partitioning $1 \mathrm{p}$ and $2 \mathrm{p}$, respectively. As discussed previously, the qubit mean-field $\left(\Psi_{\mathrm{QMF}}\right)$ and qubit coupled cluster $\left(\Psi_{\mathrm{QCC}}\right)$ wavefunctions are considered, with the only difference that $\Psi_{\mathrm{QCC}}$ is a very accurate but not exact ground state wavefunction for $\mathrm{LiH}$ (thus there is a small but non-zero variance of the $\hat{H}_{\mathrm{LiH}}$ on $\left.\Psi_{\text {QCC }}\right)$. Details on the generation of these functions can be found in ref. 25. Variances across different partitionings do not differ appreciably and the main advantage of the MFpartitioning schemes is in the reduction of the number of terms that need to be measured.

\section{Conclusions}

We have introduced and studied a new method for partitioning of the qubit Hamiltonian in the VQE approach to the electronic structure problem. The main idea of our approach is to find Hamiltonian fragments that have eigenstates consisting of single products of one- and two-qubit wavefunctions. The most general criterion for identifying such Hamiltonian fragments was derived for the first time. Once such fragments are found the total wavefunction of the system can be measured on a fragment Hamiltonian in a single pass of $N$ single-qubit measurements intertwined with one- and two-qubit rotations that are defined on-the-fly from results of previous qubit measurements. The main gain from such a reformulation is a decrease of separately measured Hamiltonian fragments. Indeed, illustrations on simple molecular systems $\left(\mathrm{H}_{2}\right.$ and $\left.\mathrm{LiH}\right)$ show three- and five-fold reductions of the number of terms that are needed to be measured with respect to the conventional scheme.

In the process of deriving our partitioning procedure, we discovered criteria for eigenstate factorability for an arbitrary Hamiltonian acting on $N$ distinguishable particles. Our criteria involve search for few-body operators commuting with the Hamiltonian of interest. Even though the criteria for factorability are exact, realistic molecular Hamiltonians do not satisfy them in general. Therefore, we needed to introduce a heuristic partitioning procedure (greedy algorithm) that splits the system Hamiltonian to fragments that have factorable eigenstates. Even though the procedure does not guarantee the absolutely optimal partitioning to the smallest number of terms, it does not produce more terms than the number of qubit-wise commuting sub-sets.

Interestingly, when one is restricted with single-qubit measurements, the commutation property of two multi-qubit operators $\hat{A}$ and $\hat{B}$ has nothing to do with the ability to measure them together (see Table 2). This seeming contradiction with the laws of quantum mechanics arises purely from a hardware restriction that one can measure a single qubit at a time. On the other hand, qubit-wise commutativity is still a sufficient but not necessary condition for single-qubit measurability. Removing the single-qubit measurement restriction in the near future will not make our scheme obsolete but rather would allow us to skip the single-particle level. For example, if two-qubit measurements will be available, one can look for two-qubit operators commuting with the Hamiltonian and integrate out pairs of qubits to define next measurable twoqubit operators.

The current approach can address difficulties arising in the exploration of the excited state via minimization of variance

$$
E(\omega)=\min _{\theta}\left\langle\Psi(\theta)\left|(\hat{H}-\omega)^{2}\right| \Psi(\theta)\right\rangle .
$$

One of the largest practical difficulties is in an increasing number of terms that are required to be measured in eqn (26). Combining some of these terms using the current methodology can reduce the number of needed measurements.

A similar problem with a growing number of terms arises if one would like to obtain the true quantum uncertainty of the measurements for a partitioned Hamiltonian, it requires measuring all covariances between all parts. Ignoring covariances by assuming measurement independence can lead to incorrect estimation of the true uncertainty, both under- and over-estimation are possible.

From the hardware standpoint, the new scheme requires modification of the single-qubit measurement protocol, where measurement results for some qubits will define unitary rotations of other qubits before their measurement, so-called feedforward measurement. This type of measurement has already been implemented in quantum computers based on superconducting $^{27}$ and photonic ${ }^{19,28,29}$ qubit architectures in the context of measurement-based quantum computing. ${ }^{15,16}$ Thus we hope that the new method will become the method of choice for quantum chemistry on a quantum computer in the near future.

\section{Appendix A: factorization conditions for the Hamiltonian eigenstates}

Here we prove that the condition given in the main text for a $N$ qubit Hamiltonian to be in the MF class is actually a necessary and sufficient condition, and hence is a criterion. We will split the proof into two parts: (1) If the Hamiltonian has $N$ oneparticle operators satisfying the reduction chain, its eigenfunctions can be written as products (sufficiency); (2) if all the Hamiltonian eigenfunctions are in a product form then it will have $N$ commuting one-particle operators defined by the reduction scheme (necessity).

(1) Proof of sufficiency: if there exist $N$ one-particle operators commuting with a set of reduced Hamiltonians it is straightforward to check that a product of eigenstates of these operators is an eigenstate of the Hamiltonian. Note that any nontrivial one-qubit operator has a non-degenerate spectrum, therefore, there is no degree of freedom related to rotation within a degenerate subspace. The choice of the first eigenstate of the first operator $\left(\hat{O}_{1}\right)$ can define the form of next one-particle operators and their eigenstates. 
(2) Proof of necessity: for the $N$-particle eigenstate $\Psi(1, \ldots N)$ to have a product form it is necessary for the Hamiltonian to have eigenstates of the $\phi_{1}(1) \Phi(2, \ldots N)$ form, where $\phi_{1}(1)$ and $\Phi(2, \ldots N)$ are some arbitrary functions from Hilbert spaces of qubit 1 and $N-1$ qubits. The latter form is an eigenstate of an operator of the form $\hat{O}_{1} \otimes I_{N-1}$, where $I_{N-1}$ is an identity operator and $\hat{O}_{1}$ is an operator for which $\phi_{1}(1)$ is an eigenfunction. Then, if the Hamiltonian and $\hat{O}_{1} \otimes I_{N-1}$ share the eigenstates they must commute. This commutation is equivalent to $\left[\hat{H}, \hat{O}_{1}\right]=0$. The same logic can be applied to $\Phi(2, \ldots N)$ because the next necessary condition for the total eigenfunction of the Hamiltonian to be in a product form is that $\Phi(2, \ldots N)=$ $\phi_{2}(2) \tilde{\Phi}(3, \ldots N)$, this gives rise to another commuting operator $\hat{O}_{2}$ whose eigenfunction is $\phi_{2}$. It is important to note though that $\hat{O}_{2}$ does not need to commute with $\hat{H}$ but only with its reduced version $H_{N-1}=\left\langle\phi_{1}|\hat{H}| \phi_{1}\right\rangle$. This chain can be continued until we reach the end of the variable list.

\section{Many-particle commuting operator extension}

Similarly if we can find an $M$-particle operator $\hat{O}$ commuting with $\hat{H}$ then, because of the theorem on commuting operators, there is a common set of eigenfunctions. With multi-qubit operators one needs to be careful because they can have a degenerate spectrum. In the case of the non-degenerate spectrum of $\hat{O}$ the common eigenstates have the factorized form $\Psi(1, \ldots N)=\Phi(1, \ldots M) \chi(M+1, \ldots N)$, which serves as a solid ground for the discussion in the main text. In the degenerate case, the most general form of a common eigenstate is $\Psi(1, \ldots N)=\sum C_{I} \Phi_{I}(1, \ldots M) \chi_{I}(M+1, \ldots N)$, where $\hat{O} \Phi_{I}(1, \ldots \mathrm{M})$ $=\lambda \Phi_{I}(1, \ldots M), I=1, \ldots k$. In this case, the important question becomes whether the Hamiltonian allows for the eigenstates to be single product states,

$$
\Psi(1, \ldots N)=\left[\sum_{I} C_{I} \Phi_{I}(1, \ldots M)\right] \chi(M+1, \ldots N),
$$

or not? To answer this question one needs to construct a reduced matrix operator within the degenerate subspace $\left\{\Phi_{I}(1, \ldots M)\right\}$

$$
\hat{H}_{I J}^{(N-M)}=\left\langle\Phi_{I}|\hat{H}| \Phi_{J}\right\rangle
$$

where integration is done over the first $M$ variables. If there exists $\Phi_{I}$ for which $\hat{H}_{I J}^{(N-M)}=0$ where $J \neq I$ then $\Psi(1, \ldots N)=$ $\Phi(1, \ldots M) \chi(M+1, \ldots N)$ will be an eigenfunction of the Hamiltonian. For all $\Phi_{I}(1, \ldots M)$ eigenfunctions to form product states, all off-diagonal elements of $\hat{H}_{I J}^{(N-M)}$ must be zero. There is one more possibility for the factorized eigenstates, if the reduced matrix operator has the particular form

$$
\hat{H}_{I J}^{(N-M)}=h_{I J} \hat{H}^{(N-M)} \text {, }
$$

where $h_{I J}$ are elements of a constant matrix and $\hat{H}^{(N-M)}$ is a single reduced operator acting on $N-M$ variables. Note that for doing this analysis one needs to be able to obtain only eigenstates of $\hat{O}$. This is presumably an easier procedure since $M<N$.
Thus, in the degenerate case, having a product form is not guaranteed and therefore, one may be able to obtain the unitary transformation unentangling qubits only in the described two cases. Yet, finding the commuting operator $\hat{O}$ is a necessary condition for the existence of an unentangling unitary transformation.

\section{Appendix B: illustration of the mean- field partitioning procedure}

To illustrate the MF partitioning procedure with a nontrivial example let us consider the model Hamiltonian whose partitioning gives rise to the scheme in Fig. 3

$$
\begin{aligned}
\hat{H}= & 3 \hat{x}_{1} \hat{x}_{2} \hat{x}_{3}+\hat{x}_{1} \hat{x}_{2} \hat{y}_{3}+5 \hat{x}_{1} \hat{x}_{2} \hat{z}_{3}+5 \hat{x}_{1} \hat{y}_{2} \hat{x}_{3}+7 \hat{x}_{1} \hat{y}_{2} \hat{z}_{3} \\
& +3 \hat{x}_{1} \hat{z}_{2} \hat{x}_{3}+\hat{x}_{1} \hat{z}_{2} \hat{y}_{3}+5 \hat{x}_{1} \hat{z}_{2} \hat{z}_{3}+6 \hat{y}_{1} \hat{x}_{2} \hat{x}_{3}+2 \hat{y}_{1} \hat{x}_{2} \hat{y}_{3} \\
& +10 \hat{y}_{1} \hat{x}_{2} \hat{z}_{3}+10 \hat{y}_{1} \hat{y}_{2} \hat{x}_{3}+14 \hat{y}_{1} \hat{y}_{2} \hat{z}_{3}+6 \hat{y}_{1} \hat{z}_{2} \hat{x}_{3}+2 \hat{y}_{1} \hat{z}_{2} \hat{y}_{3} \\
& +10 \hat{y}_{1} \hat{z}_{2} \hat{z}_{3}+3 \hat{z}_{1} \hat{x}_{2} \hat{x}_{3}+\hat{z}_{1} \hat{x}_{2} \hat{y}_{3}+5 \hat{z}_{1} \hat{x}_{2} \hat{z}_{3}+5 \hat{z}_{1} \hat{y}_{2} \hat{x}_{3} \\
& +7 \hat{z}_{1} \hat{y}_{2} \hat{z}_{3}+3 \hat{z}_{1} \hat{z}_{2} \hat{x}_{3}+\hat{z}_{1} \hat{z}_{2} \hat{y}_{3}+5 \hat{z}_{1} \hat{z}_{2} \hat{z}_{3}
\end{aligned}
$$

To assess whether the partitioning of $\hat{H}$ is possible based on qubit $k=1$ we rewrite the Hamiltonian as

$$
\hat{H}=\hat{x}_{1} \hat{h_{x}}+\hat{y}_{1} \hat{h_{y}}+\hat{z}_{1} \hat{h_{z}}
$$

where

$$
\begin{aligned}
& \hat{h_{x}}=3 \hat{x}_{2} \hat{x}_{3}+\hat{x}_{2} \hat{y}_{3}+5 \hat{x}_{2} \hat{z}_{3}+5 \hat{y}_{2} \hat{x}_{3}+7 \hat{y}_{2} \hat{z}_{3}+3 \hat{z}_{2} \hat{x}_{3} \\
& +\hat{z}_{2} \hat{y}_{3}+5 \hat{z}_{2} \hat{z}_{3} \\
& \hat{h_{y}}=6 \hat{x}_{2} \hat{x}_{3}+2 \hat{x}_{2} \hat{y}_{3}+10 \hat{x}_{2} \hat{z}_{3}+10 \hat{y}_{2} \hat{x}_{3}+14 \hat{y}_{2} \hat{z}_{3} \\
& +6 \hat{z}_{2} \hat{x}_{3}+2 \hat{z}_{2} \hat{y}_{3}+10 \hat{z}_{2} \hat{z}_{3} \\
& \hat{h_{z}}=3 \hat{x}_{2} \hat{x}_{3}+\hat{x}_{2} \hat{y}_{3}+5 \hat{x}_{2} \hat{z}_{3}+5 \hat{y}_{2} \hat{x}_{3}+7 \hat{y}_{2} \hat{z}_{3}+3 \hat{z}_{2} \hat{x}_{3} \\
& +\hat{z}_{2} \hat{y}_{3}+5 \hat{z}_{2} \hat{z}_{3}
\end{aligned}
$$

Each $\hat{h}_{x, y, z}$ is transformed into a vector. For example

$$
\overrightarrow{h_{x}}=\left[\begin{array}{llllllll}
3 & 1 & 5 & 5 & 7 & 3 & 1 & 5
\end{array}\right]^{T}
$$

in the basis $\left\{\hat{x}_{2} \hat{x}_{3}, \hat{x}_{2} \hat{y}_{3}, \hat{x}_{2} \hat{z}_{3}, \hat{y}_{2} \hat{x}_{3}, \hat{y}_{2} \hat{z}_{3}, \hat{z}_{2} \hat{x}_{3}, \hat{z}_{2} \hat{y}_{3}, \hat{z}_{2} \hat{z}_{3}\right\} . S_{1}$ is obtained as $A_{1}^{\dagger} A_{1}$, where $A_{1}=\left[\vec{h}_{x} \vec{h}_{y} \vec{h}_{z}\right]$. Diagonalizing of $S_{1}$ gives one non-zero eigenvalue $d$ and a corresponding eigenvector $\vec{v}$. The dimensionality of the $S_{1}$ kernel is $2, l(1)=2$, and it implies collinearity of $\vec{h}_{x, y, z}$ (Fig. 2a). Performing similar analysis for $S_{2}$ and $S_{3}$, one can find $l(2)=l(3)=1$ (see Fig. 3). Therefore, we rewrite the Hamiltonian as $\hat{H}=\hat{h}(2,3) \hat{O}_{1}$, where

$$
\begin{aligned}
\hat{O}_{1}= & 0.408248 \hat{x}_{1}+0.816497 \hat{y}_{1}+0.408248 \hat{z}_{1} \\
\hat{h(2,3)=} & 7.34847 \hat{x}_{2} \hat{x}_{3}+2.44949 \hat{x}_{2} \hat{y}_{3}+12.2474 \hat{x}_{2} \hat{z}_{3} \\
& +12.2474 \hat{y}_{2} \hat{x}_{3}+17.1464 \hat{y}_{2} \hat{z}_{3}+7.34847 \hat{z}_{2} \hat{x}_{3} \\
& +2.44949 \hat{z}_{2} \hat{y}_{3}+12.2474 \hat{z}_{2} \hat{z}_{3}
\end{aligned}
$$

$\hat{O}_{1}$ and $\hat{h}(2,3)$ were obtained through a linear combination of $\left\{\hat{x}_{1}, \hat{y}_{1}, \hat{z}_{1}\right\}$ and $\left\{\hat{h}_{x}, \hat{h}_{y}, \hat{h}_{z}\right\}$ with coefficients from the eigenvector $\vec{v}$.

As the next step, we consider $\hat{h}(2,3)$, it can be partitioned based on either qubit $k=2$ or $k=3$. Both qubits have the same 
values of $l(k)=1$ and $\vec{h}_{x, y, z}$ are in a single plane (Fig. 2b). Here, we choose arbitrarily $k=2$, diagonalizing $S_{2}$ leads to two nonzero eigenvalues $\left(d_{1}, d_{2}\right)$ and corresponding eigenvectors $\left(\vec{v}_{1}, \vec{v}_{2}\right)$. Following the procedure, $\hat{h}(2,3)$ decomposes to

$$
\hat{h}(2,3)=\hat{h}^{\prime}(3) \hat{O}_{2}^{\prime}+\hat{h}^{\prime \prime}(3) \hat{O}_{2}^{\prime \prime},
$$

where

$$
\begin{gathered}
\hat{O}_{2}^{\prime}=0.507019 \hat{x}_{2}-0.697039 \hat{y}_{2}+0.507019 \hat{z}_{2} \\
\hat{h^{\prime}(3)}=-1.08532 \hat{x}_{3}+2.48388 \hat{y}_{3}+0.467647 \hat{z}_{3} \\
\hat{O}_{2}^{\prime \prime}=0.492881 \hat{x}_{2}+0.717033 \hat{y}_{2}+0.492881 \hat{z}_{2} \\
\hat{h}^{\prime \prime}(3)=16.0257 \hat{x}_{3}+2.41461 \hat{y}_{3}+24.3676 \hat{z}_{3} .
\end{gathered}
$$

The single-qubit operators $\left\{\hat{O}_{2}^{\prime}, \hat{O}_{2}^{\prime \prime}\right\}$ and their complements $\left\{\hat{h}^{\prime}, \hat{h}^{\prime \prime}\right\}$ were obtained taking linear combinations of $\left\{\hat{x}_{2}, \hat{y}_{2}, \hat{z}_{2}\right\}$ and $\left\{\hat{h}_{x}, \hat{h}_{y}, \hat{h}_{z}\right\}$ with coefficients from the eigenvectors $\left(\vec{v}_{1}, \vec{v}_{2}\right)$, respectively.

The complexity of a single step of the MF partitioning procedure is polynomial with the number of qubits. In each step we need to evaluate the $l(k)$ function for each of the qubits present. Evaluation of the $l(k)$ function requires building the corresponding overlap matrix $S_{k}$, which involves inner products between columns of $A_{k}$ matrices. Since the length of $A_{k}$ columns $\left(\bar{h}_{x, y, z}\right)$ scales as $N^{4}$ at most (this is the scaling of the total number of terms in the Hamiltonian), the construction of $S_{k}$ scales as $N^{4}$ as well. Thus funding $l(k)$ functions for all qubits in general has $O\left(N^{5}\right)$ scaling.

\section{Appendix C: Hamiltonian details}

\section{$\mathrm{H}_{2}$ molecule}

One- and two-electron integrals in the canonical restricted Hartree-Fock (RHF) molecular orbital basis for $R(\mathrm{H}-\mathrm{H})=1.5 \AA$ were used in the BK transformation to produce the corresponding qubit Hamiltonian. Spin-orbitals were alternating in the order $\alpha, \beta, \alpha, \ldots$. The explicit expression for the BK qubit Hamiltonian is given in the ESI. $\dagger$

\section{LiH molecule}

A qubit Hamiltonian for $R(\mathrm{Li}-\mathrm{H})=3.2 \AA$ distance was generated using the parity fermion-to-qubit transformation. ${ }^{30}$ Spinorbitals were arranged as "first all alpha then all beta" in the fermionic form; since there are 3 active molecular orbitals in the problem, this leads to a 6-qubit Hamiltonian. Further details on the Hamiltonian are given in the ESI. $\dagger$

\section{Conflicts of interest}

There are no conflicts of interest to declare.

\section{Acknowledgements}

A.F.I. is grateful to D. Segal, P. Brumer, R. Kapral, J. Schofield, J. R. McClean, D. F. James, and I. Dhand for useful discussions.
A.F.I. acknowledges financial support from the Natural Sciences and Engineering Research Council of Canada.

\section{Notes and references}

$\ddagger$ Here, we use the notation $\left| \pm_{\sigma}\right\rangle_{n}$ for the $n^{\text {th }}$ qubit eigenstates of a $\sigma$ one-particle operator with \pm 1 eigenvalues.

$\S$ To simplify the notation we use freedom in qubit enumeration and assume that we work with the qubit enumeration that follows the described reductive sequence.

1 A. Peruzzo, J. McClean, P. Shadbolt, M.-H. Yung, X.-Q. Zhou, P. J. Love, A. Aspuru-Guzik and J. L. O'Brien, A variational eigenvalue solver on a photonic quantum processor, Nat. Commun., 2014, 5, 4213.

2 J. R. McClean, J. Romero, R. Babbush and A. Aspuru-Guzik, The theory of variational hybrid quantum-classical algorithms, Am. J. Phys., 2016, 18, 023023.

3 D. Wecker, M. B. Hastings and M. Troyer, Progress towards practical quantum variational algorithms, Phys. Rev. A, 2015, 92, 042303.

4 J. Olson; Y. Cao; J. Romero; P. Johnson; P.-L. DallaireDemers; N. Sawaya; P. Narang; I. Kivlichan; M. Wasielewski and A. Aspuru-Guzik Quantum Information and Computation for Chemistry. arXiv.org 2017.

5 S. McArdle; S. Endo; A. Aspuru-Guzik; S. Benjamin and X. Yuan Quantum computational chemistry. arXiv.org 2018.

$6 \mathrm{P}$. Jordan and E. Wigner, Über das Paulische Äquivalenzverbot, Z. Phys., 1928, 47, 631-651.

7 A. Aspuru-Guzik, A. D. Dutoi, P. J. Love and M. Head-Gordon, Simulated Quantum Computation of Molecular Energies, Science, 2005, 309, 1704-1707.

8 S. B. Bravyi and A. Y. Kitaev, Fermionic Quantum Computation, Ann. Phys., 2002, 298, 210-226.

9 J. T. Seeley, M. J. Richard and P. J. Love, The Bravyi-Kitaev transformation for quantum computation of electronic structure, J. Chem. Phys., 2012, 137, 224109.

10 A. Tranter, S. Sofia, J. Seeley, M. Kaicher, J. McClean, R. Babbush, P. V. Coveney, F. Mintert, F. Wilhelm and P. J. Love, The Bravyi-Kitaev transformation: Properties and applications, Int. J. Quantum Chem., 2015, 115, 1431.

11 K. Setia and J. D. Whitfield, Bravyi-Kitaev Superfast simulation of fermions on a quantum computer, ArXiv eprints, 2017.

12 V. Havlíček, M. Troyer and J. D. Whitfield, Operator locality in the quantum simulation of fermionic models, Phys. Rev. A, 2017, 95, 032332.

13 A. Kandala, A. Mezzacapo, K. Temme, M. Takita, M. Brink, J. M. Chow and J. M. Gambetta, Hardware-efficient variational quantum eigensolver for small molecules and quantum magnets, Nature, 2017, 549, 242-246.

14 I. G. Ryabinkin, S. N. Genin and A. F. Izmaylov, Constrained Variational Quantum Eigensolver: Quantum Computer Search Engine in the Fock Space, J. Chem. Theory Comput., 2019, 15, 249-255.

15 M. A. Nielsen, Cluster-state quantum computation, Rep. Math. Phys., 2006, 57, 147-161. 
16 A. Mantri, T. F. Demarie and J. F. Fitzsimons, Universality of quantum computation with cluster states and (X, Y)-plane measurements, Sci. Rep., 2017, 7, 1-7.

17 T. Helgaker; P. Jorgensen and J. Olsen Molecular Electronicstructure Theory, Wiley, 2000.

18 F. Albarrán-Arriagada, G. A. Barrios, M. Sanz, G. Romero, L. Lamata, J. C. Retamal and E. Solano, One-way quantum computing in superconducting circuits, Phys. Rev. A, 2018, 97, 032320.

19 R. Prevedel, P. Walther, F. Tiefenbacher, P. Böhi, R. Kaltenbaek, T. Jennewein and A. Zeilinger, High-speed linear optics quantum computing using active feedforward, Nature, 2007, 445, 65-69.

20 D. Ristè and L. DiCarloDigital feedback in superconducting quantum circuits. arXiv.org 2015.

21 R. Barends, J. Kelly, A. Megrant, D. Sank, E. Jeffrey, Y. Chen, Y. Yin, B. Chiaro, J. Mutus, C. Neill, P. O'Malley, P. Roushan, J. Wenner, T. C. White, A. N. Cleland and J. M. Martinis, Coherent Josephson Qubit Suitable for Scalable Quantum Integrated Circuits, Phys. Rev. Lett., 2013, 111, 080502.

22 M. Körber; O. Morin; S. Langenfeld; A. Neuzner; S. Ritter and G. Rempe, Decoherence-protected memory for a singlephoton qubit. arXiv.org 2017.

23 S. Bravyi; J. M. Gambetta; A. Mezzacapo and K. Temme, Tapering off qubits to simulate fermionic Hamiltonians. arXiv.org 2017.

24 C. Hempel, C. Maier, J. Romero, J. McClean, T. Monz, H. Shen, P. Jurcevic, B. P. Lanyon, P. Love, R. Babbush,
A. Aspuru-Guzik, R. Blatt and C. F. Roos, Quantum Chemistry Calculations on a Trapped-Ion Quantum Simulator, Phys. Rev. X, 2018, 8, 031022.

25 I. G. Ryabinkin, T.-C. Yen, S. N. Genin and A. F. Izmaylov, Qubit coupled cluster method: A systematic approach to quantum chemistry on a quantum computer, J. Chem. Theory Comput., 2018, 14, 6317-6326.

26 I. G. Ryabinkin, S. N. Genin and A. F. Izmaylov, Relation between fermionic and qubit mean fields in the electronic structure problem, J. Chem. Phys., 2018, 149, 214105.

27 R. Vijay, C. Macklin, D. H. Slichter, S. J. Weber, K. W. Murch, R. Naik, A. N. Korotkov and I. Siddiqi, Stabilizing Rabi oscillations in a superconducting qubit using quantum feedback, Nature, 2012, 490, 77-80.

28 L. M. Procopio, A. Moqanaki, M. Araújo, F. Costa, I. A. Calafell, E. G. Dowd, D. R. Hamel, L. A. Rozema, v. Brukner and P. Walther, Experimental superposition of orders of quantum gates, Nat. Commun., 2015, 6, 1-6.

29 C. Reimer, S. Sciara, P. Roztocki, M. Islam, L. R. Cortés, Y. Zhang, B. Fischer, S. Loranger, R. Kashyap, A. Cino, S. T. Chu, B. E. Little, D. J. Moss, L. Caspani, W. J. Munro, J. Azaña, M. Kues and R. Morandotti, High-dimensional one-way quantum processing implemented on d-level cluster states, Nat. Phys., 2019, 15, 148-153.

30 M. A. Nielsen The Fermionic canonical commutation relations and the Jordan-Wigner transform. School of Physical Sciences The University of Queensland 2005. 